\title{
COMPORTAMIENTO POSTCOSECHA DE FRUTOS DE AGUACATE 'HASS’ AFECTADO POR TEMPERATURA Y ATMÓSFERA MODIFICADA CON MICROPERFORADO
}

\section{POSTHARVEST BEHAVIOR OF 'HASS' AVOCADO FRUIT AFFECTED BY TEMPERATURE AND MICROPERFORATED BASED-MODIFIED ATMOSPHERE}

\author{
C. Cristóbal Espinosa-Cruz ${ }^{1}$, Salvador Valle-Guadarrama ${ }^{1 \star}$, Ma. Carmen Ybarra-Moncada $^{1}$ \\ y M. Teresa Martínez-Damián²
}

\begin{abstract}
${ }^{1}$ Departamento de Ingeniería Agroindustrial y ${ }^{2}$ Departamento de Fitotecnia, Universidad Autónoma Chapingo (UACh). Km. 38.5 Carr. México-Texcoco. 56230, Chapingo. México, México.
\end{abstract}

${ }^{*}$ Autor para correspondencia (svalleg@taurus.chapingo.mx).

\section{RESUMEN}

El fruto de aguacate (Persea americana Mill.) 'Hass' puede manejarse con refrigeración y atmósferas modificadas (AM) en postcosecha, pero las AM pueden causar metabolismo fermentativo por disponibilidad insuficiente de $\mathrm{O}_{2}$, lo que hace necesario controlar el intercambio gaseoso en el envase; esto último puede atenderse con microperforado. El objetivo del trabajo fue evaluar el efecto de baja temperatura y AM con envase microperforado en el comportamiento postcosecha de frutos de aguacate 'Hass'. Se usaron recipientes de tereftalato de polietileno (PET) de $1.87 \mathrm{~L}$ con $0,2,4,6$ y 8 orificios de $200 \mu \mathrm{m}$ en las paredes, con lotes de tres frutos y peso total de 550.0 ( \pm 34.2) g, donde al variar el número de perforaciones se pudo controlar la concentración de $\mathrm{O}_{2}$ en los envases. El uso de microperforado con 4 y 2 orificios fue adecuado para manejar los frutos a 18 y $5{ }^{\circ} \mathrm{C}$, con $\mathrm{O}_{2}$ en concentración de 3.5 y $4.0 \%$, respectivamente, pues se redujo pérdida de peso, velocidad de ablandamiento, velocidad de cambio de color y la actividad fermentativa, con relación a un almacenamiento en aire normal.

Palabras clave: Persea americana, atmósfera modificada, microperforado, postcosecha.

\section{SUMMARY}

The 'Hass' avocado (Persea americana Mill.) fruit can be handled with refrigeration and modified atmosphere packaging (MAP), but MAP may cause fermentative metabolism due to low $\mathrm{O}_{2}$ availability. Thus, gas exchange must be controlled, and this can be achieved with microperforation. The effect of using low temperature and microperforated package on postharvest behavior of 'Hass' avocado fruit was evaluated. polyethylene terephthalate (PET) containers of $1.87 \mathrm{~L}$, with $0,2,4,6$, and 8 holes of $200 \mu \mathrm{m}$ on the walls were used with batches of three fruits and total weight of $550.0( \pm 34.2) \mathrm{g}$. By modification of the number of perforations, $\mathrm{O}_{2}$ concentration in packages was controlled. The microperforated-MAP technique with 4 and 2 microholes was convenient to handle fruit at 18 and $5{ }^{\circ} \mathrm{C}$, with concentrations of $\mathrm{O}_{2}$ of 3.5 and $4.0 \%$, respectively, because it caused reduction in weight loss, softening rate, color change rate, and fermentative activity, compared to open air storage.

Index words: Persea americana, modified atmosphere, microperforation, postharvest.

\section{INTRODUCCIÓN}

El aguacate (Persea americana Mill.) es una especie de gran importancia socioeconómica en México, país que tiene $25 \%$ de la superficie mundial cultivada y genera hasta 33 $\%$ de la producción total de fruta (Téliz y Marroquín, 2007). El fruto de aguacate se consume principalmente en fresco y para conservarlo se usa primordialmente refrigeración (Perez et al., 2004). Sin embargo, hay regiones productoras sin infraestructura para aplicar este método, lo que crea la necesidad de explorar otras estrategias. Al respecto, se han estudiado procedimientos para controlar los efectos del etileno (Osuna et al., 2005; Ochoa-Ascencio et al., 2009), se han aplicado recubrimientos poliméricos (Aguilar-Méndez et al., 2008) y se ha demostrado el potencial de uso de sistemas de atmósfera modificada (AM) para alargar la vida útil en postcosecha (Hertog et al., 2003).

Una AM usa recipientes plásticos como envase, donde mediante la acción combinada de la respiración y la permeación gaseosa a través del polímero, el $\mathrm{O}_{2}$ se reduce y el $\mathrm{CO}_{2}$ se incrementa, lo que causa alteración de diversos procesos fisiológicos y alarga la vida de anaquel (Mangaraj et al., 2009). Un problema frecuente en esta técnica es la falta de control de la permeabilidad a gases del envase, lo que puede causar reducción excesiva de $\mathrm{O}_{2}$ y alta concentración de $\mathrm{CO}_{2}$, con riesgo de inducir metabolismo fermentativo, cuyos productos, acetaldehído y etanol, pueden dañar el material conservado e inducir malos olores y sabores (Rojas-Graü et al., 2009). En el caso de aguacate 'Hass', el fruto no resiste ambientes con concentración de $\mathrm{O}_{2}$ menor a 2 $\%$ sin que haya fermentación (Hertog et al., 2003), y se ha demostrado que en condiciones de $20^{\circ} \mathrm{C}$ tal metabolismo ocurre cuando el $\mathrm{O}_{2}$ está presente en concentración de 5 $\%$ o menor en el ambiente (Valle-Guadarrama et al., 2013).

Hasta ahora no hay trabajos enfocados al control del intercambio gaseoso en las paredes del envase plástico en el 
manejo de frutos de aguacate 'Hass' en AM, pero con otros productos se ha mostrado que al usar microperforaciones con diámetro menor a $200 \mu \mathrm{m}$ se pueden controlar las concentraciones de $\mathrm{O}_{2}$ y CO 2 en el entorno (Makino et al., 2008; Monroy-Gutiérrez et al., 2013). Por ello, el objetivo de este trabajo fue evaluar el efecto de baja temperatura y de AM con envase microperforado en el comportamiento postcosecha de frutos de aguacate 'Hass'.

\section{MATERIALES Y MÉTODOS}

\section{Material vegetal}

Se usaron frutos de aguacate 'Hass' en madurez fisiológica, con $22.2( \pm 0.4) \%$ de materia seca, $192.2( \pm 10.1) \mathrm{g}$ de peso, $53.6( \pm 2.0) \mathrm{N}$ de firmeza y $120^{\circ}\left( \pm 1.8^{\circ}\right)$ de ángulo de matiz en cáscara, que fueron cosechados en marzo de 2012 en Uruapan, Michoacán, México ( $19^{\circ} 25^{\prime} \mathrm{N} ; 102^{\circ} 03^{\prime}$ O; $1620 \mathrm{msnm})$.

\section{Evaluación del efecto del microperforado sobre las concentraciones de $\mathrm{O}_{2} \mathrm{y} \mathrm{CO}_{2}$}

Para las AM se usaron envases de tereftalato de polietileno (PET) de $1.87 \mathrm{~L}$ de capacidad. Se formaron cinco lotes de seis envases cada uno, los cuales se nombraron $\mathrm{M}_{0}, \mathrm{M}_{2}$, $\mathrm{M}_{4}, \mathrm{M}_{6} \mathrm{y} \mathrm{M}_{8}, \mathrm{y}$ contuvieron tres frutos de aguacate con peso total de $550( \pm 34.2) \mathrm{g}$. En los envases de los cuatro últimos lotes se hicieron 2, 4, 6 y 8 microperforaciones de $200 \mu \mathrm{m}$, respectivamente, y los de $M_{0}$ se dejaron sin perforar. Tres envases de cada lote se colocaron a temperatura ambiente $\left(18 \pm 2{ }^{\circ} \mathrm{C}\right)$ y los tres restantes a $5( \pm 1)^{\circ} \mathrm{C}$, donde se permitió acondicionamiento térmico por $5 \mathrm{~h}$ antes de ser cerrados. En cada envase se monitoreó la concentración de $\mathrm{O}_{2} \mathrm{y}$ $\mathrm{CO}_{2}$ a intervalos de $4 \mathrm{~h}$ durante $48 \mathrm{~h}$, con apoyo de un cromatógrafo de gases (Varian Star ${ }^{\circledR}, 3400 \mathrm{CX}$, USA) equipado con columna Chrompack Poraplot- $\mathrm{Q}{ }^{\circledR}$, detector por conductividad térmica y detector por ionización de flama, que operó a 80,150 y $170{ }^{\circ} \mathrm{C}$ en la columna, inyector y detectores, respectivamente, con presión manométrica de columna de $158.5 \mathrm{kPa}$. Con los datos se ajustaron modelos de regresión con la forma $\left[y_{\mathrm{O}_{2} / \mathrm{CO}_{2}}=y_{0}+a(1-\exp (-b t))\right]$, donde $y_{\mathrm{O}_{2} / \mathrm{CO}_{2}}$ es concentración (\%) $\mathrm{de}_{2} \mathrm{O}_{2} \mathrm{CO}_{2}$ al tiempo transcurrido $(t$, h), y $y_{0}(\%), a(\%)$ y $b\left(\mathrm{~h}^{-1}\right)$ son constantes de regresión. Los modelos se usaron para evaluar las concentraciones de $\mathrm{O}_{2} \mathrm{y}$ $\mathrm{CO}_{2}$ alcanzadas en condición de régimen estacionario $\left(y_{\infty}\right)$ y éstas sirvieron para elegir un nivel de microperforado en cada condición térmica para tener concentraciones de $\mathrm{O}_{2}$ sin riesgo de metabolismo fermentativo.

\section{Evaluación del comportamiento postcosecha de frutos}

Se formaron 96 lotes de tres frutos cada uno. De ellos,
48 se colocaron en recipientes de tereftalato de polietileno (PET) de 1.87 L con el grado de microperforación seleccionado en la fase anterior $\left(M_{2}\right.$ para refrigeración y $M_{4}$ para ambiente). Los 48 lotes restantes se destinaron a manejo en aire natural (AN). Cada lote se consideró una unidad experimental (UE). Del total de recipientes la mitad se colocó a $18( \pm 2)^{\circ} \mathrm{C}$ y $76 \%$ de humedad relativa (HR) y la otra mitad a $5( \pm 1){ }^{\circ} \mathrm{C}$ y $89 \% \mathrm{HR}$. El día de instalación del experimento, y posteriormente cada 3 ó $4 \mathrm{~d}$, hasta completar 21 y $28 \mathrm{~d}$, respectivamente, se retiraron tres UE de cada tipo de manejo, para evaluar concentración de $\mathrm{O}_{2} \mathrm{y} \mathrm{CO}_{2}$ en los recipientes, pérdida de peso, color de cáscara, firmeza de pulpa y contenido de metabolitos anaerobios (acetaldehído y etanol).

La pérdida de peso se expresó en porcentaje respecto al peso inicial de los frutos. El color se evaluó con un colorímetro Hunter Lab ${ }^{\circledR}$ (Mini Scan XE Plus 45/0-L, USA) y se expresó como ángulo de matiz. La firmeza se expresó en newtons $(\mathrm{N})$, como el promedio de tres determinaciones hechas en los frutos con un analizador de textura (TAXT2i ${ }^{\circledR}$, Stable Microsystems, UK), equipado con un aditamento esférico de $5 \mathrm{~mm}$ de diámetro, el cual deformó la pulpa hasta $2.5 \mathrm{~mm}$ a tasa de $3 \mathrm{~mm} \mathrm{~s}^{-1}$.

Para medir contenido de metabolitos anaerobios se adaptó el método de Davis y Chase (1969), con muestras de 10 g de pulpa colocadas en viales de $37 \mathrm{~mL}$, cuyo espacio de cabeza se muestreó con alícuotas de $1 \mathrm{~mL}$ después de una incubación en baño maría por $10 \mathrm{~min}$, las cuales se inyectaron en el cromatógrafo de gases descrito antes, que operó a 160,170 y $170{ }^{\circ} \mathrm{C}$ en la columna, inyector y detectores, respectivamente. La determinación se estos compuestos se auxilió con soluciones de acetaldehído en concentraciones

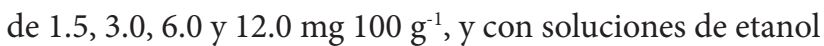
en concentraciones de 10, 20, 40, 70, 100 y $140 \mathrm{mg} 100 \mathrm{~g} \mathrm{~g}^{-1}$, las cuales se inyectaron al equipo también en alícuotas de $1 \mathrm{~mL}$ para servir de referente a través de la construcción de curvas tipo. En adición, con base en la estequiometría de la ruta de la fermentación alcohólica (Taiz y Zeiger, 2006), las producciones molares de acetaldehído y etanol se sumaron para expresar contenido de metabolitos anaerobios $\left(\mu \mathrm{mol} 100 \mathrm{~g}^{-1}\right)$. Finalmente, las concentraciones de $\mathrm{O}_{2} \mathrm{y}$ $\mathrm{CO}_{2}$ se evaluaron con el mismo equipo de cromatografía con muestras tomadas de los envases, lo cual se auxilió con estándares en concentración de 2.01 y $4.98 \%$ para $\mathrm{O}_{2}$ y de 5.01 y $50.0 \%$ para $\mathrm{CO}_{2}$ (Praxair Technology Inc., México).

\section{Análisis de datos}

La fase de evaluación del efecto del microperforado sobre las concentraciones de $\mathrm{O}_{2}$ y $\mathrm{CO}_{2}$ en los envases se realizó conforme a un arreglo factorial $2 \times 5$ bajo un diseño completamente al azar. La temperatura $\left(\mathrm{T}: 18\right.$ y $5{ }^{\circ} \mathrm{C}$ ) y el 
nivel de microperforado ( $M: M_{0}, M_{2}, M_{4}, M_{6}$ y $M_{8}$ ) fueron los factores de variación. Por otro lado, la fase de estudio de frutos en postcosecha se hizo con un arreglo que usó también a $\mathrm{T}\left(18\right.$ y $\left.5{ }^{\circ} \mathrm{C}\right)$ y el tipo de ambiente (A: AM y aire normal) como factores de variación, y la evaluación se hizo en ocho momentos en el tiempo $(\Phi)$. En ambas fases los datos se sometieron a análisis de varianza y rutinas de comparación de medias (Tukey, 0.05). En la segunda fase, el efecto de T y $\Phi$ se determinó con base en el error del efecto de interacción T $x \Phi$.

\section{RESULTADOS Y DISCUSIÓN}

\section{Concentración de $\mathrm{O}_{2}$ y $\mathrm{CO}_{2}$ en el interior de envases}

Los estudios de AM de $48 \mathrm{~h}$ con distintos grados de microperforación mostraron que la concentración de $\mathrm{O}_{2}$ disminuyó y la de $\mathrm{CO}_{2}$ se incrementó con el tiempo en forma logarítmica, con cambios rápidos al inicio y con tendencia tangencial hacia un valor constante al final (Figura 1). Los datos se ajustaron bien a modelos de la forma $\left[y_{O_{2} / \mathrm{CO}_{2}}=y_{0}\right.$ $+a(1-\exp (-b t))]$, con coeficientes de determinación $\left(R^{2}\right)$ mayores a 0.94 , excepto en el comportamiento de $\mathrm{O}_{2}$ de los tratamientos $\mathrm{M}_{6}$ y $\mathrm{M}_{8}$ a $5{ }^{\circ} \mathrm{C}$ donde, por tener un cambio total pequeño, el equilibrio se alcanzó rápidamente y la tendencia se alejó del modelo logarítmico. El coeficiente de cambio $b\left(\mathrm{~h}^{-1}\right)$, que expresa la modificación de concentración de $\mathrm{O}_{2}$ o $\mathrm{CO}_{2}$ por unidad de tiempo y por unidad de gradiente de concentración entre el interior y el exterior de los recipientes, tuvo valores entre 0.052 y $0.196 \mathrm{~h}^{-1}$ para $\mathrm{O}_{2}$, y entre 0.078 y $0.52 \mathrm{~h}^{-1}$ para $\mathrm{CO}_{2}$. En virtud de que un equilibrio mayor a $90 \%$ tiene magnitud de $[(1 / b) \ln 10]$ (Brosnan y Sun, 2001), tal condición se alcanzó en los recipientes entre 11 y $44 \mathrm{~h}$ para $\mathrm{O}_{2}$ y entre 4 y $29 \mathrm{~h}$ para $\mathrm{CO}_{2}$.

Por otro lado, la suma $\left(y_{0}+a\right)$ coincidió con la asíntota de las curvas de cambio de concentración y correspondió con el valor de concentración alcanzada $\left(y_{\infty}\right)$ tras la estabilización (Cuadro 1), lo cual fue similar a lo observado por Monroy-Gutiérrez et al. (2013) en sistemas de AM microperforados que se usaron con huitlacoche (Ustilago maydis; hongos comestibles que crecen en mazorcas de maíz, Zea mays). El análisis de efectos mayores del diseño factorial mostró que este valor $y_{\infty}$, llamado concentración de régimen estacionario, se afectó por la temperatura (Cuadro 2), de forma que para $\mathrm{O}_{2}$ y $\mathrm{CO}_{2}$ tuvo valores promedio de $3.6 \mathrm{y}$ $22.1 \%$ a $18{ }^{\circ} \mathrm{C}$, respectivamente, los cuales fueron distintos $(\mathrm{P} \leq 0.05)$ a los alcanzados a $5{ }^{\circ} \mathrm{C}(9.0$ y $13.2 \%)$, con diferencias significativas honestas (DSH) de 0.33 y $0.37 \%$, respectivamente. También hubo efecto significativo del grado de microperforación, aunque el análisis de varianza reportó interacción significativa entre este factor y la temperatura ( $\mathrm{P}$ $\leq 0.05$, Cuadro 2). En ambas condiciones térmicas, cuanto mayor fue el número de perforaciones, mayores y menores $(\mathrm{P} \leq 0.05)$ fueron las concentraciones $y_{\infty}$ alcanzadas por el $\mathrm{O}_{2}$ y el $\mathrm{CO}_{2}$, respectivamente (Cuadro 1), lo cual confirmó lo postulado por Makino et al. (2008), en el sentido que el microperforado constituye una manera efectiva de controlar la concentración de gases en envases de sistemas de AM.

A $18{ }^{\circ} \mathrm{C}$ los niveles de microperforado $\mathrm{M}_{0}$ y $\mathrm{M}_{2}$ causaron que el $\mathrm{O}_{2}$ fuera menor al valor de $2 \%$ en los envases (Cuadro 1, Figura 1) el cual, según Hertog et al. (2003) es la concentración mínima de este gas que tolera el fruto de aguacate 'Hass' para no desarrollar metabolismo fermentativo. Una situación similar ocurrió en el tratamiento $\mathrm{M}_{0}$ aplicado a $5{ }^{\circ} \mathrm{C}$, lo que indica que ninguna de estas opciones es adecuada para manejar este fruto en postcosecha, por el riesgo de causar fermentación. En tal contexto, como mejores tratamientos a 18 y a $5^{\circ} \mathrm{C}$ se eligió a los que usaron cuatro y dos microperforaciones en el envase $\left(M_{4}\right.$ y $\left.M_{2}\right)$, donde el $\mathrm{O}_{2}$ se estabilizó en concentraciones de 3.5 y $4.0 \%$, respectivamente.

\section{Comportamiento postcosecha de frutos en refrigeración y AM basada en microperforado}

Concentraciones de $\mathrm{O}_{2} \mathrm{y} \mathrm{CO}_{2}$. Durante la evaluación de frutos por 21 a 28 d, la concentración de $\mathrm{O}_{2}$ se redujo y la de $\mathrm{CO}_{2}$ se incrementó en los envases de $\mathrm{AM}$ en los primeros $5 \mathrm{~d}$ (Figura $2 \mathrm{~A}$ ) y esta modificación fue significativa $(\mathrm{P} \leq$ 0.05; Cuadro 3). También hubo efecto notorio de la temperatura, de manera que el $\mathrm{O}_{2}$ se equilibró con $3.53 \%( \pm 0.50$ $\%)$ a $18{ }^{\circ} \mathrm{C}$ y con $5.26 \%( \pm 0.67 \%)$ a $5{ }^{\circ} \mathrm{C}(\mathrm{DSH}=0.37 \%)$, en tanto que el $\mathrm{CO}_{2}$ se estabilizó en $23.22 \%( \pm 0.93 \%)$ a 18 ${ }^{\circ} \mathrm{C}$ y en $15.58 \%( \pm 0.69 \%)$ a $5{ }^{\circ} \mathrm{C}(\mathrm{DSH}=0.56 \%)$ (Figura $2 \mathrm{~A}$ ). Estos valores fueron similares a los que se predijeron en la primera fase del trabajo y ello confirmó que con 4 y 2 microperforaciones a 18 y $5{ }^{\circ} \mathrm{C}$, respectivamente, se logra que el $\mathrm{O}_{2}$ se equilibre con más de $2 \%$, que es la tolerancia del fruto a bajo nivel de $\mathrm{O}_{2}$ (Hertog et al., 2003).

Producción de metabolitos anaerobios. La temperatura (T) y el tipo de ambiente (A) afectaron significativamente la producción de metabolitos anaerobios, pero no así el tiempo $(\Phi)$, aunque la interacción de los primeros con este último fue significativa $(\mathrm{P} \leq 0.05$; Cuadro 3$)$. A $18{ }^{\circ} \mathrm{C}$ el manejo en aire causó producción promedio de $330.8 \mu \mathrm{mol}$ $100 \mathrm{~g}^{-1}$, con un incremento súbito a partir del día 16 de almacenamiento, lo cual contrastó $(\mathrm{P} \leq 0.05)$ con la producción de $132.8 \mu \mathrm{mol} 100 \mathrm{~g}^{-1}$ observada en el material de $\mathrm{AM}$, donde no hubo grandes variaciones (Figura 2B), lo que sugiere que los frutos de la condición térmica alta experimentaron alteración en su comportamiento metabólico. Se ha demostrado que en frutos de aguacate 'Hass' puede observarse la manifestación combinada de metabolismos aeróbico (oxidativo) y anaeróbico (fermentativo), derivado 
$18^{\circ} \mathrm{C}$
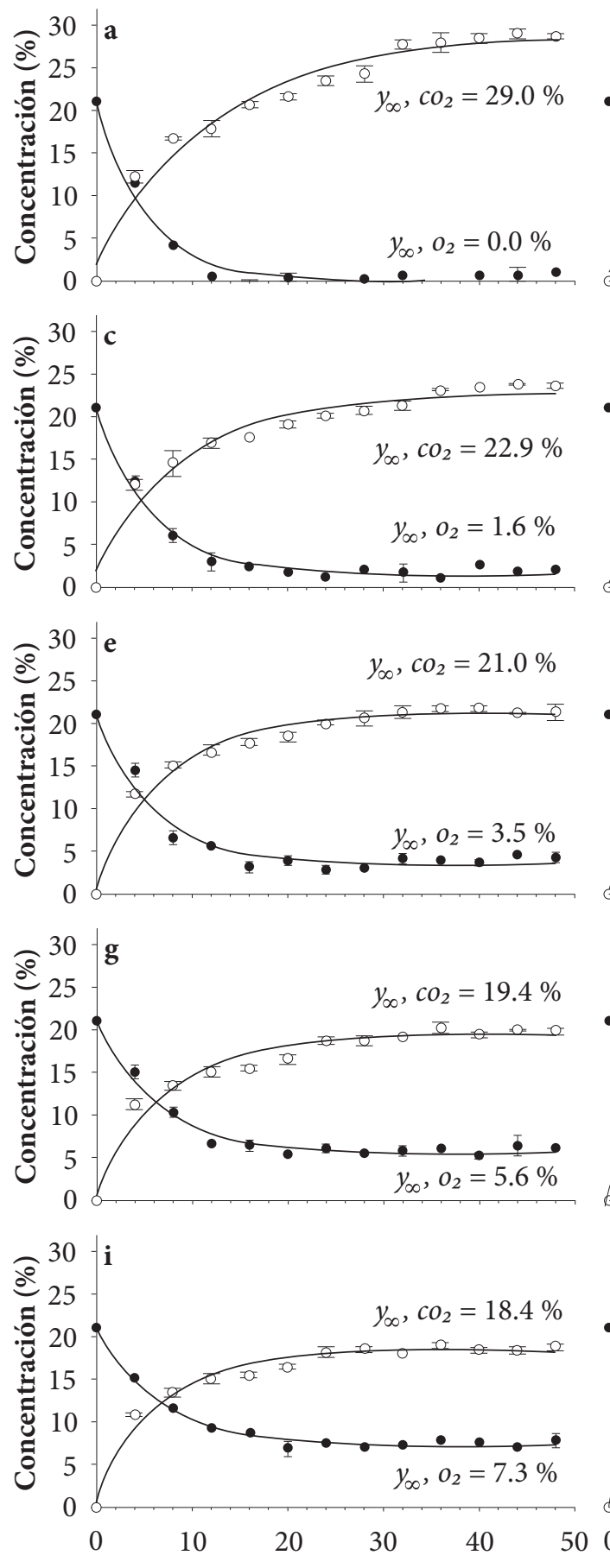

Tiempo (h) $5^{\circ} \mathrm{C}$
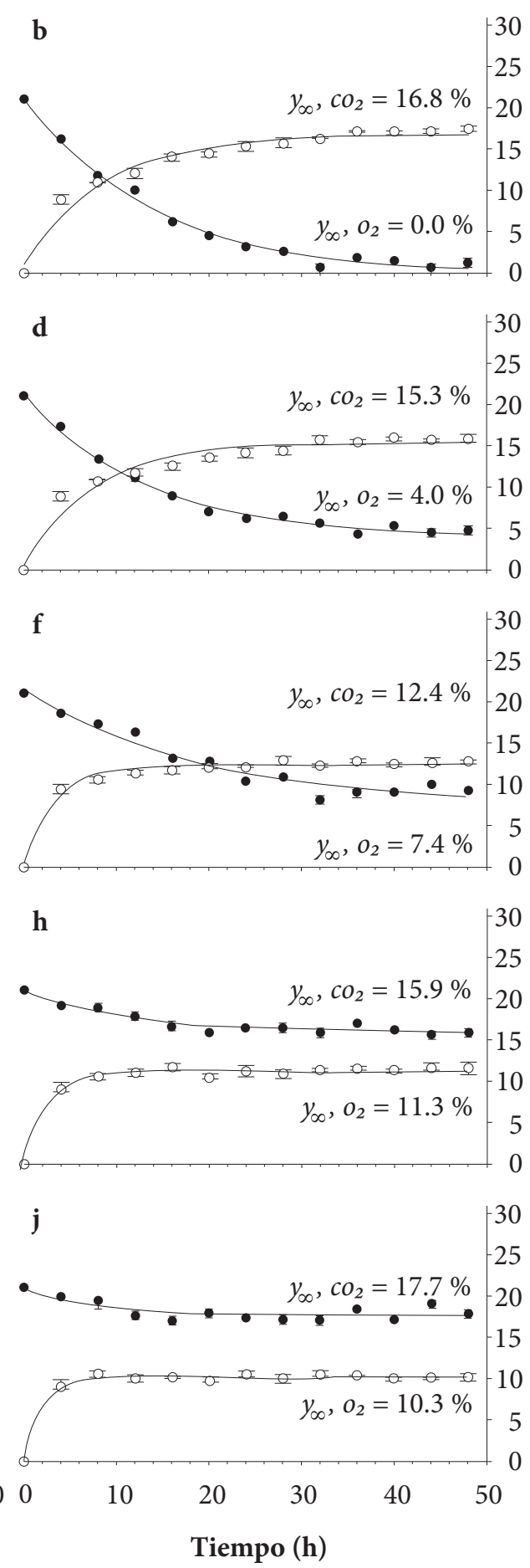

Figura 1. Cinéticas de cambio de concentración de $\mathrm{O}_{2}(\bullet)$ y $\mathrm{CO}_{2}(\circ)$ a $18{ }^{\circ} \mathrm{C}$ (izquierda) y $5{ }^{\circ} \mathrm{C}$ (derecha) durante la fase de estabilización de sistemas de atmósfera modificada en recipientes de PET de $1.87 \mathrm{~L}$ con número de microperforaciones en envases igual a $0(\mathrm{a}, \mathrm{b}), 2(\mathrm{c}, \mathrm{d}), 4(\mathrm{e}, \mathrm{f}), 6(\mathrm{~g}, \mathrm{~h})$ y $8(\mathrm{i}, \mathrm{j})$. Los símbolos $(\bullet, \circ)$ representan la media de tres repeticiones, las barras indican error estándar y las líneas corresponden a ajustes obtenidos mediante procedimientos de regresión no lineal. Los valores de $y_{\infty, o_{2}}$ y $y_{\infty, c_{2}}$ corresponden a las concentraciones de $\mathrm{O}_{2}$ y $\mathrm{CO}_{2}$ alcanzadas en los recipientes tras la fase de estabilización. 
Cuadro 1. Constantes de regresión relacionadas con el modelo $\left\{y=y_{0}+a[1-\exp (-b t)]\right\}$. La suma $\left[y=y_{0}+a\right]$ expresa concentración de $\mathrm{O}_{2} \mathrm{OCO}_{2}$ en la condición de equilibrio alcanzada en los envases de atmósfera modificada.

\begin{tabular}{|c|c|c|c|c|c|c|c|c|c|c|}
\hline \multirow{3}{*}{ Perforaciones } & $y_{0}(\%)$ & $a(\%)$ & $b\left(\mathrm{~h}^{-1}\right)$ & $R^{2}$ & $y_{\infty}(\%)$ & $y_{0}(\%)$ & $a(\%)$ & $b\left(\mathrm{~h}^{-1}\right)$ & $R^{2}$ & $y_{\infty}(\%)$ \\
\hline & \multicolumn{5}{|c|}{$\mathrm{O}_{2}$} & \multicolumn{5}{|c|}{$\mathrm{CO}_{2}$} \\
\hline & \multicolumn{10}{|c|}{$18^{\circ} \mathrm{C}$} \\
\hline 0 & 21.51 & -21.53 & 0.196 & 0.97 & $0.00 \mathrm{e}^{\dagger}$ & 2.19 & 26.79 & 0.078 & 0.95 & $28.98 \mathrm{a}$ \\
\hline 2 & 21.36 & -19.76 & 0.181 & 0.97 & $1.60 \mathrm{~d}$ & 1.69 & 21.19 & 0.107 & 0.94 & $22.88 \mathrm{~b}$ \\
\hline 4 & 21.63 & -18.09 & 0.179 & 0.95 & $3.54 \mathrm{c}$ & 0.91 & 20.11 & 0.145 & 0.96 & $21.02 \mathrm{c}$ \\
\hline 6 & 21.46 & -15.81 & 0.162 & 0.96 & $5.65 \mathrm{~b}$ & 1.14 & 18.24 & 0.135 & 0.95 & $19.38 \mathrm{~d}$ \\
\hline 8 & 21.17 & -13.90 & 0.153 & 0.97 & $7.27 \mathrm{a}$ & 0.71 & 17.57 & 0.161 & 0.96 & $18.28 \mathrm{~d}$ \\
\hline \multirow{2}{*}{ DSH } &.---- &.---- &.---- &.---- & 1.06 &.---- & --.-- &.---- &.---- & 1.42 \\
\hline & \multicolumn{10}{|c|}{$5^{\circ} \mathrm{C}$} \\
\hline 0 & 21.35 & -21.51 & 0.073 & 0.98 & $0.00 \mathrm{e}$ & 1.01 & 15.83 & 0.116 & 0.96 & $16.84 \mathrm{a}$ \\
\hline 2 & 21.40 & -17.37 & 0.078 & 0.98 & $4.03 \mathrm{~d}$ & 0.92 & 14.42 & 0.135 & 0.94 & $15.34 \mathrm{~b}$ \\
\hline 4 & 21.52 & -14.14 & 0.052 & 0.94 & $7.38 \mathrm{c}$ & 0.14 & 12.23 & 0.305 & 0.96 & $12.37 \mathrm{c}$ \\
\hline 6 & 21.06 & -5.12 & 0.093 & 0.81 & $15.94 \mathrm{~b}$ & 0.04 & 11.22 & 0.387 & 0.95 & $11.26 \mathrm{~cd}$ \\
\hline 8 & 21.28 & -3.62 & 0.151 & 0.59 & $17.66 \mathrm{a}$ & 0.03 & 10.18 & 0.520 & 0.96 & $10.21 \mathrm{~d}$ \\
\hline DSH &.---- & $--\cdot--$ & --.-- &.---- & 1.24 &.---- &.---- &.---- &.---- & 1.18 \\
\hline
\end{tabular}

$†$ Letras diferentes en la columna de $y_{\infty}$ indican diferencias significativas con la diferencia significativa honesta (DSH; Tukey, 0.05$) . R^{2}$ es coeficiente de determinación

Cuadro 2. Valores percentiles de la distribución de Fisher $\left(\mathrm{F}_{0.05}\right)$ con $(\alpha=0.05)$ y valores de $F$ correspondientes al análisis de varianza de la evaluación de $48 \mathrm{~h}$ del efecto de la temperatura $(\mathrm{T})$ y el microperforado $(\mathrm{M})$ sobre las concentraciones de régimen estacionario de $\mathrm{O}_{2} \mathrm{yCO}_{2}\left(y_{\infty}, o_{2}, y_{\infty}, \mathrm{Co}_{2}\right)$ alcanzadas en recipientes de atmósfera modificada.

\begin{tabular}{lcccc}
\hline \multirow{2}{*}{ F.V. } & $\mathrm{gl}$ & $\mathrm{F}_{0.05}$ & \multicolumn{2}{c}{ Valores de F } \\
\cline { 4 - 5 } & & & $y_{\mathrm{o}_{2}}$ & $y_{\mathrm{o}, \mathrm{CO}_{2}}$ \\
$\mathrm{~T}$ & 1 & 4.35 & $1175.4^{*}$ & $2517.0^{*}$ \\
$\mathrm{M}$ & 4 & 2.87 & $885.3^{*}$ & $303.5^{*}$ \\
$\mathrm{~T} \times \mathrm{M}$ & 4 & 2.87 & $184.7^{*}$ & $21.6^{*}$ \\
Error & 20 &.---- &.---- &.---- \\
$\mathrm{CV}(\%)$ &.---- &.---- & 6.77 & 2.75 \\
\hline
\end{tabular}

F.V. = factor de variación; $\mathrm{CV}=$ coeficiente de variación; $\mathrm{gl}=$ grados de libertad; $y_{\mathrm{os}_{2}} \mathrm{y} y_{\mathrm{Qq}_{\mathrm{CO}}}=$ variable de concentración de $\mathrm{O}_{2} \mathrm{y} \mathrm{CO}_{2}$ en recipientes; ${ }^{*}=$ efecto significativo a $\mathrm{P} \leq 0.05$.

de una insuficiente provisión de $\mathrm{O}_{2}$, lo que propicia el incremento de la producción de compuestos de la fermentación (Valle-Guadarrama et al., 2013). Al respecto, en frutos de papaya (Carica papaya), que tienen también comportamiento climatérico y procesos de ablandamiento en algún grado similares a aguacate, se ha encontrado que esto ocurre por modificaciones en difusividad gaseosa, integridad de membranas y características histológicas, derivadas de reducción de firmeza y senescencia (Pereira et al., 2009).

En cambio, a $5{ }^{\circ} \mathrm{C}$ no hubo diferencia en la producción de estos metabolitos entre los dos tipos de manejo (aire y AM) y los valores promedio fueron de 30.2 y $26.4 \mu \mathrm{mol} 100$ $\mathrm{g}^{-1}$, respectivamente (Figura 2B), lo que muestra que la baja temperatura reduce de forma importante la actividad metabólica y compensa el bajo suministro de $\mathrm{O}_{2}$ que puede producirse en un manejo en AM. En tal sentido se confirmó que las condiciones de $\mathrm{AM}\left(\mathrm{M}_{4}\right)$ a $18^{\circ} \mathrm{C}$ y las dos condiciones de manejo a $5{ }^{\circ} \mathrm{C}$ (aire y $\mathrm{M}_{2}$ ) presentaron características que pueden favorecer el manejo de los frutos en postcosecha, pues la actividad fermentativa que causaron fue baja.

Pérdida de peso. Esta variable también fue afectada por $\mathrm{T}$ y A, con interacción significativa de estos factores con el tiempo de almacenamiento ( $P \leq 0.05$; Cuadro 3$)$. En ambas temperaturas el manejo en aire causó los cambios más 

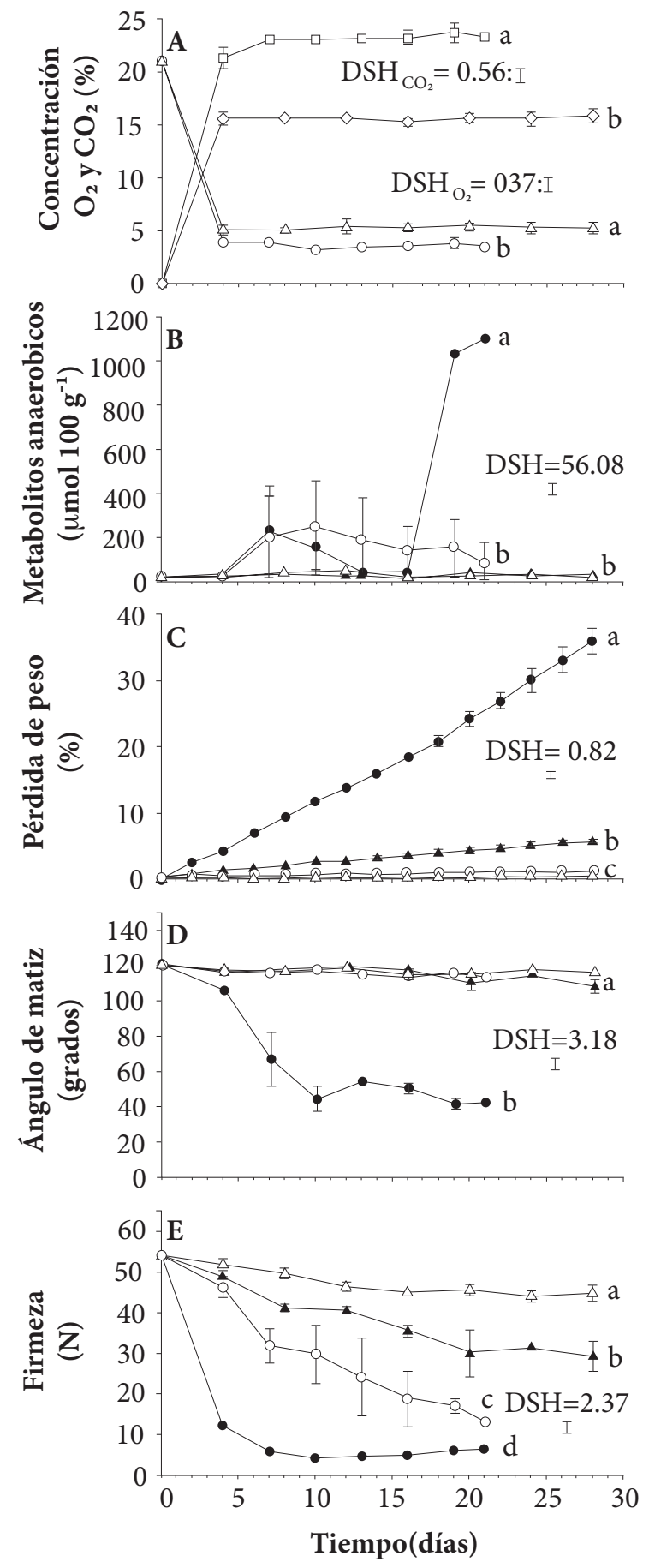

Figura 2. Concentraciones de $\mathrm{O}_{2}$ y $\mathrm{CO}_{2}(\mathrm{~A})$, producción de metabolitos anaerobios $(B)$, variación de pérdida de peso (C), ángulo de matiz (D) y firmeza (E) en frutos de aguacate 'Hass' almacenados en atmósfera modificada (AM) a 18 y $5^{\circ} \mathrm{C}$. Símbolos en recuadros de $\mathrm{B}$ a E: $(\bullet$, aire normal a $\left.18^{\circ} \mathrm{C}\right),\left(\mathrm{O}, \mathrm{AM}\right.$ con 4 microperforaciones a $\left.18^{\circ} \mathrm{C}\right)$, $\left(\Delta\right.$, aire normal a $\left.5^{\circ} \mathrm{C}\right),(\triangle, \mathrm{AM}$ con 2 microperforaciones a $\left.5{ }^{\circ} \mathrm{C}\right)$. Símbolos en recuadro $\mathrm{A}:\left(\circ, \triangle: \mathrm{O}_{2}\right.$ en $\mathrm{AM}$ a $18 \mathrm{y}$ $\left.5{ }^{\circ} \mathrm{C}\right),\left(\square, \diamond: \mathrm{CO}_{2}\right.$ en AM a 18 y $\left.5^{\circ} \mathrm{C}\right)$. DSH es diferencia

significativa honesta (Tukey, 0.05). Cada valor representa la media de tres observaciones y las barras indican error estándar. Letras minúsculas distintas indican diferencia significativa general.

grandes de peso, que alcanzaron pérdidas de $35.9 \%$ a $18^{\circ} \mathrm{C}$ y de $5.7 \%$ a $5{ }^{\circ} \mathrm{C}$, que contrastaron $(\mathrm{P} \leq 0.05)$ con el valor promedio menor a $1.0 \%$ observado en los materiales manejados en AM, sin diferencia en éstos por efecto del tiempo o temperatura (Figura 2C). La pérdida de peso en postcosecha se atribuye principalmente a transpiración causada por un déficit de presión de vapor del producto con relación a su entorno, el cual, por la baja humedad relativa se incrementó en la condición de aire natural y mayor temperatura. De hecho, el uso de películas plásticas y refrigeración se encuentra entre las estrategias sugeridas para disminuir la pérdida de peso (Ben-Yehoshua y Rodov, 2003), pues contribuyen a reducir el déficit mencionado, lo cual explica el efecto observado a $5{ }^{\circ} \mathrm{C}$ y AM en el presente trabajo.

Ángulo de matiz. El color de cáscara es un atributo que se relaciona de manera directa con el grado de madurez en frutos de aguacate 'Hass' (Hertog et al., 2003; Cox et al., 2004; Osuna et al., 2005). En el presente trabajo esta variable se afectó por la temperatura y por el tipo de ambiente, con interacción significativa con el tiempo de almacenamiento ( $\mathrm{P} \leq 0.05$; Cuadro 3 ), lo cual ocurrió porque el manejo refrigerado, ya sea en aire o en $\mathrm{AM}$, y el de $\mathrm{AM}$ a $18^{\circ} \mathrm{C}$, hizo que el color se mantuviera prácticamente sin cambios, en tanto que con manejo en aire a $18^{\circ} \mathrm{C}$ se observaron cambios del valor inicial de $120^{\circ}$ hasta $42.9^{\circ}$ al cumplirse $21 \mathrm{~d}$ de almacén(Figura 2D) lo cual constituye una evidencia del efecto positivo del manejo en baja temperatura y AM en el retraso de la maduración.

Firmeza. El ablandamiento es el rasgo más característico del proceso de maduración de un fruto de aguacate 'Hass' y se considera un criterio de referencia para evaluar la vida postcosecha potencial (Ochoa-Ascencio et al., 2009). En el presente trabajo la firmeza se afectó por los tres factores evaluados ( $\mathrm{T}$, A y $\Phi)$, entre los cuales hubo interacción significativa ( $\mathrm{P} \leq 0.05$; Cuadro 3$)$. En todos los casos hubo reducción notable de la firmeza con el tiempo $(\mathrm{P} \leq 0.05)$, con la mayor velocidad de cambio en el manejo en aire a $18{ }^{\circ} \mathrm{C}$, seguida por los manejos de $\mathrm{AM}$ a $18^{\circ} \mathrm{C}$, aire a $5^{\circ} \mathrm{C}$ y $\mathrm{AM}$ a $5^{\circ} \mathrm{C}$, en ese orden, con diferencia significativa entre ellos (Figura 2E).

En el tratamiento de aire a $18^{\circ} \mathrm{C}$, los frutos resultaron blandos en $7 \mathrm{~d}$, al cambiar la firmeza de 53.6 a $5.7 \mathrm{~N}$ en la condición de consumo, en tanto que con el manejo en AM a la misma temperatura la firmeza era aún de $16.9 \mathrm{~N}$ al cumplirse $19 \mathrm{~d}$ de almacenamiento. Por tanto, el manejo en AM con microperforado puede ser una alternativa adecuada para zonas 
Cuadro 3. Valores percentiles de la distribución de Fisher $\left(\mathrm{F}_{0.05}\right)$ con $(\alpha=0.05)$ y valores de $\mathrm{F}$ correspondientes al análisis de varianza de la evaluación del efecto de la temperatura ( $\mathrm{T}: 18$ ó $\left.5{ }^{\circ} \mathrm{C}\right)$, el tipo de ambiente (A: aire o AM) y el tiempo ( de almacenamiento) sobre el comportamiento postcosecha de frutos de aguacate 'Hass'.

\begin{tabular}{|c|c|c|c|c|c|c|c|c|}
\hline \multirow{2}{*}{ FV } & \multirow{2}{*}{ gl } & \multirow{2}{*}{$\mathrm{F}_{0.05}$} & \multicolumn{6}{|c|}{ Valores de F } \\
\hline & & & $y_{\mathrm{O}_{2}}$ & $y_{\mathrm{CO}_{2}}$ & Met-An & $\mathrm{PP}$ & $\mathrm{H}^{*}$ & Firm \\
\hline $\mathrm{T}$ & 1 & 3.98 & $36.5^{*}$ & $45.5^{*}$ & $5.9^{*}$ & $31.5^{*}$ & $25.4^{*}$ & $46.0^{*}$ \\
\hline A & 1 & 3.98 &.---- & --.-- & $5.4^{*}$ & $530.1 *$ & $178.8^{*}$ & $98.8^{*}$ \\
\hline$\Phi$ & 7 & 2.14 & $300.8^{*}$ & $25.4^{\star}$ & $1.0 \mathrm{~ns}$ & $1.9 \mathrm{~ns}$ & $1.5 \mathrm{~ns}$ & $4.2^{*}$ \\
\hline $\mathrm{T} \times \mathrm{A}$ & 1 & 3.98 &.---- & --.-- & $5.2^{*}$ & $257.8^{*}$ & $163.4^{\star}$ & $13.8^{*}$ \\
\hline $\mathrm{T} \times \Phi$ & 7 & 2.14 & $2.4^{*}$ & $12.2^{*}$ & $3.7^{*}$ & $9.4^{*}$ & $7.1^{*}$ & $7.6^{*}$ \\
\hline $\mathrm{A} \times \Phi$ & 7 & 2.14 &.---- &.---- & $3.8^{*}$ & 16.0 * & $7.4^{*}$ & $2.6^{*}$ \\
\hline Error & 71 & --.-- &.---- & --.-- &.---- &.---- &.---- & --.-- \\
\hline CV (\%) & --.-- & --.-- & 8.22 & 5.62 & 89.89 & 52.37 & 9.08 & 18.88 \\
\hline
\end{tabular}

$\mathrm{FV}$ = factor de variación; $\mathrm{CV}=$ coeficiente de variación; $\mathrm{gl}=$ grados de libertad; $y_{\mathrm{O}_{2}} \mathrm{y} y_{\mathrm{CO}_{2}}=$ concentración de $\mathrm{O}_{2}$ y $\mathrm{CO}_{2}$ en recipientes de atmósfera modificada; $\mathrm{PP}$ = pérdida de peso; Met- $\mathrm{An}=$ metabolitos anaerobios; $\mathrm{H}^{*}=$ Ángulo de matriz; Firm $=$ fírmeza; ${ }^{*}=$ efecto significativo a $\mathrm{P} \leq 0.05$.

productoras de aguacate donde no existe infraestructura de refrigeración. Por otro lado, fue evidente que el mejor tratamiento fue el que combinó refrigeración $\left(5^{\circ} \mathrm{C}\right)$ con $\mathrm{AM}$ microperforada, pues la firmeza sólo cambió de 53.6 a 44.6 $\mathrm{N}$ en $28 \mathrm{~d}$ de almacenamiento.

\section{CONCLUSIONES}

El microperforado permitió controlar la concentración de gases en los envases de atmósfera modificada (AM) de forma que con 4 orificios a $18^{\circ} \mathrm{C}$ y 2 orificios a $5{ }^{\circ} \mathrm{C}$ se logró tener 3.5 y $4.0 \%$ de concentración de $\mathrm{O}_{2}$, y 23.2 y $16.6 \%$ de $\mathrm{CO}_{2}$, respectivamente. El uso de refrigeración permitió reducir los cambios de peso, firmeza y color de los frutos de aguacate 'Hass', características que constituyen referentes importantes de la calidad de los mismos. Al combinar esta estrategia con sistemas de AM microperforada las velocidades de pérdida de peso, ablandamiento y oscurecimiento de cáscara tuvieron los valores más bajos, con baja actividad fermentativa, con lo cual se probó que la técnica de microperforado es una alternativa viable para el manejo de frutos de aguacate 'Hass' en postcosecha.

\section{BIBLIOGRAFÍA}

Aguilar-Méndez M. A., E. S. Martín-Martínez, S. A. Tomás, A. CruzOrea and M. R. Jaime-Fonseca (2008) Gelatine-starch films: physicochemical properties and their application in extending the post-harvest shelf life of avocado (Persea americana). Journal of the Science of Food and Agriculture 88:185-193.

Ben-Yehoshua S. and V. Rodov (2003) Transpiration and water stress. In: Postharvest Physiology and Pathology of Vegetables. J. A. Bartz and J. K. Brecht (eds). University of Florida. Gainesville, Florida. pp:111-159.

Brosnan T. and D. W. Sun (2001) Precooling techniques and applications for horticultural products - a review. International Journal of Refrigeration 24:154-170.
Cox K. A., T. K. McGhie, A. White and A. B. Woolf (2004) Skin colour and pigment changes during ripening of 'Hass' avocado fruit Postharvest Biology and Technology 31:287-294.

Davis P. L. and W. G. Chase (1969) Determination of alcohol in citrus juice by gas chromatographic analysis of head space. HortScience 4:117-119.

Hertog M. L. A. T. M., S. E. Nicholson and K. Whitmore (2003) The effect of modified atmospheres on the rate of quality change in 'Hass' avocado. Postharvest Biology and Technology 29:41-53.

Makino Y., S. Oshita, Y. Kawagoe and A. Tanaka (2008) Simultaneous prediction of oxygen and carbon dioxide concentrations in a perforated pouch with light red tomato fruits by a mathematical model. Transactions of the ASABE 51:559-565.

Mangaraj S., T. K. Goswami and P. V. Mahajan (2009) Applications of plastic films for modified atmosphere packaging of fruits and vegetables: a review. Food Engineering Reviews 1:133-158.

Monroy-Gutiérrez T., S. Valle-Guadarrama, T. Espinosa-Solares, M. T. Martínez-Damián and A. Pérez-López (2013) Effect of microperforation and temperature on quality of modified atmosphere packaged huitlacoche (Ustilago maydis). CyTA - Journal of Food 11:309-317.

Ochoa-Ascencio S., M. L. A. T. M. Hertog and B. M. Nicolä̈ (2009) Modelling the transient effect of 1-MCP on 'Hass' avocado softening: a Mexican comparative study. Postharvest Biology and Technology 51:62-72.

Osuna G. J. A., J. A. Beltrán and V. V. Valdivia (2005) Efecto del 1-metilciclopropeno (1-MCP) sobre el comportamiento postcosecha del aguacate 'Hass'. Revista Fitotecnia Mexicana 28:1-8.

Pereira T., A. P. S. Gomes, A. I. Goncalves, M. da Cunha, O. J. Goncalves, S. M. Gomes and H. Vargas (2009) Gas diffusion in 'Golden' papaya fruit at different maturity stages. Postharvest Biology and Technology 54:123-130.

Perez K., J. Mercado and H. Soto-Valdez (2004) Note. Effect of storage temperature on the shelf life of Hass avocado (Persea americana). Food Science and Technology International 10:73-77.

Rojas-Graï M. A., G. Oms-Oliu, R. Soliva-Fortuny and O. Martín-Belloso (2009) The use of packaging techniques to maintain freshness in fresh-cut fruits and vegetables: a review. International Journal of Food Science and Technology 44:875-889.

Taiz L. and E. Zeiger (2006) Plant Physiology (4th ed.). Sinauer Associates Inc. Publishers, USA. 650 p.

Téliz O. D. and P. F. J. Marroquín (2007) Importancia histórica y socioeconómica del aguacate. In: El Aguacate y su Manejo Integrado (Segunda Edición). D Téliz, A Mora (eds.). Ediciones 
Mundi-Prensa. México. pp:1-28.

Valle-Guadarrama S., M. Morales-Cabrera, C. B. Peña-Valdivia, B. Mora-Rodríguez, I. Alia-Tejacal, J. Corrales-García and A. Gó-
mez-Cruz (2013) Oxidative/fermentative behavior in the flesh of 'Hass' avocado fruits under natural and controlled atmosphere conditions. Food and Bioprocess Technology 6:272-282. 